PAPER

3D shape measurement of complex transparent liquid surfaces using monoscopic deformed fringe transmissometry

To cite this article: Menglin Zhong et al 2019 Meas. Sci. Technol. 30115201

View the article online for updates and enhancements. 


\title{
3D shape measurement of complex transparent liquid surfaces using monoscopic deformed fringe transmissometry
}

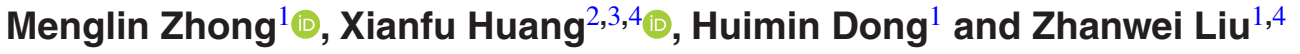 \\ ${ }^{1}$ School of Aerospace Engineering, Beijing Institute of Technology, Beijing 100081, \\ People's Republic of China \\ 2 State Key Laboratory of Nonlinear Mechanics, Institute of Mechanics, Chinese Academy of Sciences, \\ Beijing 100190, People's Republic of China \\ ${ }^{3}$ School of Engineering Science, University of Chinese Academy of Sciences, Beijing 100049, \\ People's Republic of China \\ E-mail: liuzw@bit.edu.cn and huangxf@imech.ac.cn
}

Received 11 February 2019, revised 21 May 2019

Accepted for publication 8 July 2019

Published 4 September 2019

\begin{abstract}
The measurement of transparent liquid surface topography is of great importance in many fields such as hydrodynamics of water-walking insects, liquid sloshing and flotation, etc. However, techniques for measuring these specular and rheological surfaces, especially for surfaces with floating objects, have been lacking. Here we report a monoscopic deformed fringe transmissometry for measuring complex transparent liquid surfaces with theoretical sensitivity up to $0.40 \mu \mathrm{m}$. The basic principle is to reconstruct the 3D liquid surfaces by the mathematical relations between the in-plane fringe phase-shift and the out-of-plane shape of the liquid surface. It only needs to project a single fringe pattern from the bottom of the liquid and capture a single deformed image from above. A sub-pixel sampling moiré method is proposed to process the captured fringe pattern to obtain the precise phase component and the displacement field, which is then used to reconstruct the 3D shape of the liquid surface. The discontinuous fringes in the pattern also settled by covering an extracted mask to the displacement filed. The verification test of floating 3D printed characters indicates that this new technique is robust in measuring the 3D shape of complex liquid surface. The developed technique provides a versatile tool to explore interfacial phenomena such as wetting, floating, surface wave, etc.
\end{abstract}

Keywords: sampling moiré method, phase-shifting, displacement field, fringe pattern

(Some figures may appear in colour only in the online journal)

\section{Introduction}

Measurement of transparent liquid surface topography is of very importance in both scientific and engineering fields. For example, Water Striders can walk, bounce, and even leap on liquid surface with their slender legs and without sinking into

${ }^{4}$ Author to whom any correspondence should be addressed. the water, that aroused great interest among the scientists of biology, fluids and bionic robotics fields [1-7]. To elucidate their superior locomotion on the water, a series of studies have been carried out to explore the liquid topography caused by the insects to reveal their fluid dynamics, including theoretical analysis [4] and laboratory experiments [2]. However, due to the lack of measurement for complex liquid surface morphology caused by Water Striders, the research of Water 


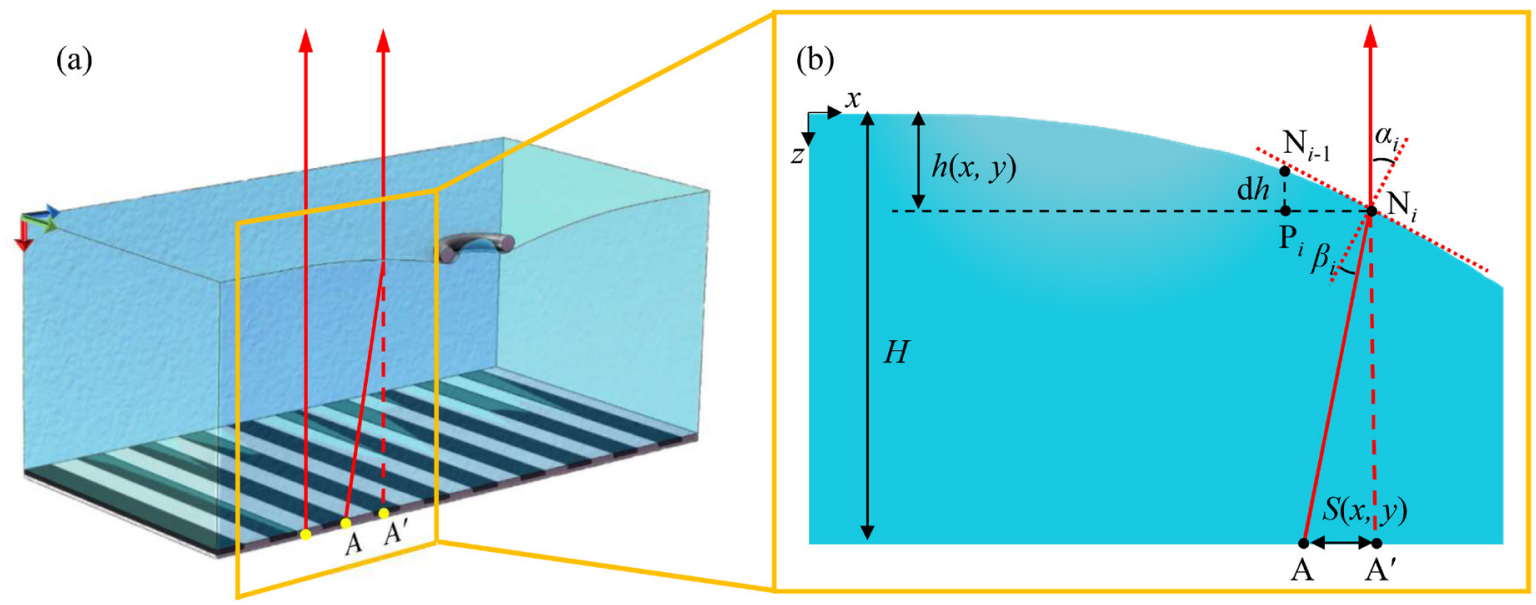

Figure 1. Schematic showing the propagation of the light as it passes through the deformed liquid surface caused by the floating object. (a) Light refraction on the surface of the transparent liquid. (b) A cross section of the deformed liquid surface showing the triangular relationship between the in-plane displacement and the surface deformation.

Striders' superior locomotion still lacks effective measurement results. Another example is the liquid sloshing in spacecrafts [7-9]. The non-linear sloshing of liquid fuels will exert a tremendous impact on the flight control of spacecrafts [10], which have led to an emphasis on the measurement of liquid surface topography in tanks. In order to solve the liquid sloshing associated with liquid surface deformation, many numerical simulation methods have been developed [11-14]. In spite of this, the real-time detection of the spacecraft tank is essential. Because the extensive applications of baffles [15] in the spacecraft tank, it is necessary to develop methods to measure the complex discontinuous liquid surface topography.

Experimental techniques for measuring of the topography of free liquid surfaces can be divided into two categories. The first one is interferometry [16, 17]. Matsuda et al [16] developed a holographic shearing interferometer that uses twin three-beam holograms to measure liquid surface deformation in real time. This technique is robust when measuring large deformations. However, for laser interferometry, the optical set-ups are very complex. In addition, this set of methods is too sensitive to out-of-plane deformation, resulting in failure in measuring large-slope liquid surfaces [16].

The second category of techniques is based primarily on the refraction or reflection of a carrier image on the liquid surface. Moisy et al [18] developed a synthetic Schlieren method to reconstruct the 3D topography of liquid surface. Huang et al [19] proposed a monoscopic fringe reflectometric system for dynamic 3D sensing of specular reflective surfaces. However, they have a limited measurements range and only suitable for small deformations. Liu et al [20-25] proposed a fringe transmission technique for measuring the deformation of large liquid surface by analyzing the in-plane deformation of transmission fringe patterns. This set of techniques have advantages of non-contact and full-field measurements, and the experimental set-up is very simple and easy to operate. However, they are not suitable for high precision deformation measurement of dynamic liquid surface, since the Fourier transform has a strain measurement limitation of 1/3 [26].
In the present paper, a monoscopic deformed fringe transmissometry combined with sub-pixel sampling moiré method and phase-shifting technique is proposed for measuring the full-field liquid surface deformation in a way with high resolution and precision. This non-contact method has the advantages of high sensitivity and measurement of discontinuous transparent liquid surfaces. In addition, it only needs to capture a single deformed fringe pattern and an undeformed one to reconstruct the liquid surface, indicating its potential for dynamic measurement. For complex liquid surface, we propose a digital mask method to achieve liquid surface topography measurements in discontinuous regions. The paper is organized as follows: the principle of the developed method is formulated in 'Method Development'. We then conducted experimental tests and verifications in 'Experiments'. The experimental results and the processing of unconnected fringe patterns are presented in full detail in 'Results and Discussion'. For the case of discontinuous liquid surface, an artificial mask is presented to deal with the inconsecutive fringes. In 'Conclusions' we give concluding remarks.

\section{Method development}

\section{Refraction of lights on a transparent liquid surface}

The basic principle of monoscopic fringe transmissometry is: when light travels to another medium, refraction occurs on the interface. When the interface is deformed, there is in-plane displacement between the position of the object below the interface seen by the eye and its actual position, also known as the virtual image principle. The interface deformation and the in-plane displacement are mathematically related and can therefore be used to reconstruct the $3 \mathrm{D}$ shape of the interface or surface.

Figure 1(a) shows the propagation of light on liquid surface. The carrier pattern such as lattices [22], fringes [23], speckles [21, 24, 25], grids [20], or dots [18], is placed at the bottom of the liquid for the measurement. First, the light of the carrier pattern is emitted from the bottom of the liquid and 


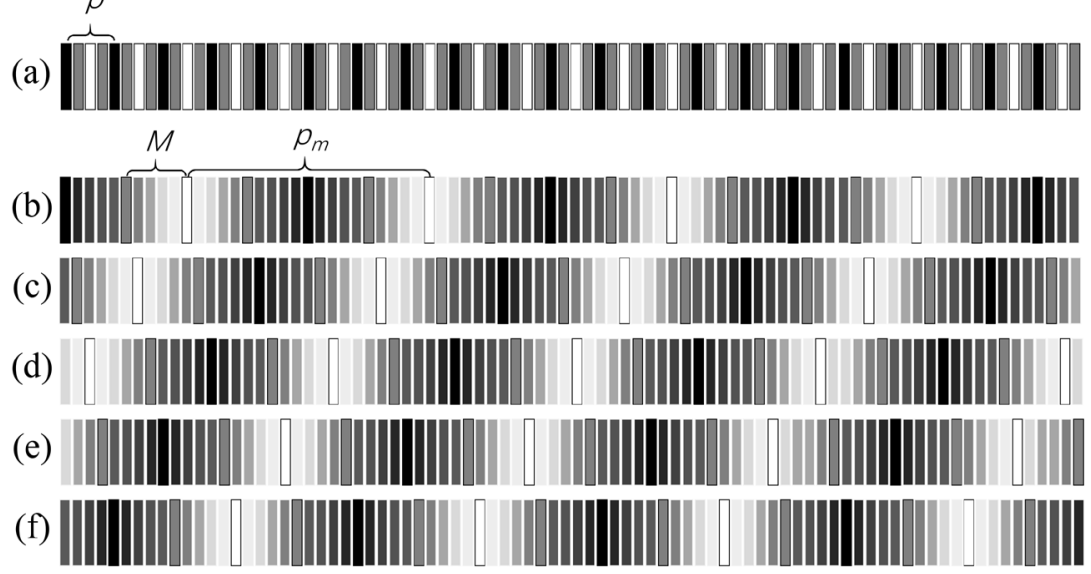

Figure 2. Principle of sampling moiré method. (a) An initial fringe specimen. (b-f) New moiré patterns generated by sampling of the fringe pattern at different points and interpolating with constant intervals.

refracts as it passes through the surface of the liquid, and then travels straight up until captured by the optical camera. When viewed from above, the virtual image of the carrier pattern is deformed as the surface of the liquid deforms. As shown in figure 1(a), if the liquid surface is deformed, the point A will move to the virtual point $A^{\prime}$ when observed from the above. There is a mathematical relationship between the morphology of the liquid surface and the deformation of the virtual image.

As shown in figure 1(b), the deformation curve of an arbitrary cross section is divided into an infinite number of small segments, wherein the connection points are denoted as $N_{1}$, $N_{2} \ldots N_{i}$. The height of each point in the curve is related to the change of height with its' neighbor point in the same curve. According to Snell's law, the triangular relationship [23] in $\triangle A^{\prime} N_{i} A$ and $\Delta N_{i} P_{i} N_{i-1}$, the three equations can be derived as:

$$
\left\{\begin{array}{l}
\sin \alpha=n_{\mathrm{w}} \cdot \sin \beta \\
\tan (\alpha-\beta)=\frac{S(x, y)}{H-h(x, y)} \\
h(x, y)=\int_{0}^{x} \tan \alpha \mathrm{d} x \\
\left.h\right|_{x=0}=0
\end{array}\right.
$$

where $n_{\mathrm{w}}$ is the refractive index of the water; $\beta$ and $\alpha$ are the incident angle and the refraction angle at a point $N$, respectively; $S(x, y)$ is the in-plane displacement of the virtual carrier patterns; $H$ is the initial height of the liquid; $h$ is the out-of-plane deformation representing the liquid surface topography.

Therefore, the height information of the liquid surface can be measured by measuring the in-plane displacement of transmission carrier pattern. In this paper, the measurement of the in-plane displacement is achieved by using the sampling moiré method combined with the phase-shift technique.

\section{The phase measurement of the fringe pattern}

To extract the in-plane displacement of transmission pattern, we employ sampling moiré method to achieve the purpose. Sampling moiré method $[27,28]$ has been wildly used to measure the displacement and strain of solid objects. The basic principle of the method is to spatially sample the grating (or fringe) pattern at constant pitch. After interpolating the image, it will form a moiré pattern. If the initial sample position of the pattern moves, the corresponding moiré fringes will also move, resulting in a series of phase-shifted moiré fringes. These moiré patterns can be analyzed using phase-shifting technique [29, 30].

Figure 2(a) shows an initial fringe pattern with a fringe spacing $p$, which was sampled at a constant pitch $M$ from the first point. After interpolation, a sampling moiré pattern with a pitch $p_{m}$ is obtained, as shown in figure 2(b). If the second point is selected as the starting point for sampling at the constant pitch $M$, a $2 \pi / M$ phase-shifted image of moire pattern can be obtained, as shown in figure 2(c) [25]. And the moiré patterns shown in figures 2(d)-(f) are selected as the third, the fourth and the fifth points of the starting point of sampling, respectively, using the same operation [25]. From a single image the multiple phase-shifted images of the moiré fringe pattern can be obtained. Therefore, a plurality of phase-shifted moiré patterns can be obtained from a single image.

The intensity of these fringe patterns can be expressed as:

$$
I_{k}(x, y)=I_{a}(x, y)+I_{b}(x, y) \cos \left[\varphi_{m}(x, y)+2 \pi \frac{k}{M}\right]
$$

where $k=(0,1,2 \ldots M-1)$ is the order of phase-shifted images and $M \in N^{*}, I_{a}(x, y)$ is the background intensity, $I_{b}(x, y)$ is the amplitude of the light intensity, and $\varphi_{m}(x, y)$ is the initial phase of moiré fringe at $(x, y)$. In general, getting information about $\varphi_{m}(x, y)$ is enough to represent the fringes without knowing the correct $I_{a}(x, y)$ and $I_{b}(x, y)$. And $\varphi_{m}(x, y)$ can be obtained after the phase-shifting processing. The wrapping phase of moiré fringe can be solved by $Q$-step phase-shifting method (here $Q=M$ ), the formula [27] is as follow:

$$
\varphi_{m}(x, y)=-\tan ^{-1} \frac{\sum_{k=0}^{M-1} I_{k}(x, y) \sin \left(k \frac{2 \pi}{M}\right)}{\sum_{k=0}^{M-1} I_{k}(x, y) \cos \left(k \frac{2 \pi}{M}\right)} .
$$

The displacement $S(x, y)$ can be obtained from the phase difference $\Delta \varphi_{m}(x, y)$ before and after deformation by using equation (4) [25]:

$$
S=p \frac{\Delta \varphi_{m}}{2 \pi}
$$


At last, combining equation (4) with (1), the relationship between the deformations of the fringes and liquid surface can obtained as:

$$
h(x, y)=H-\frac{p}{2 \pi} \Delta \varphi_{m}(x, y) \Psi\left\{\frac{\partial h(x, y)}{\partial x}\right\}
$$

where $\Psi(u)=\frac{\sqrt{n_{\mathrm{w}}^{2}+\left(n_{\mathrm{w}}^{2}-1\right) \cdot u^{2}}+u^{2}}{u \times\left\{\sqrt{n_{\mathrm{w}}^{2}+\left(n_{\mathrm{w}}^{2}-1\right) \cdot u^{2}}-1\right\}}$ and $\left.h(x, y)\right|_{x=0}=\frac{\partial h(x, y)}{\partial x}$ $\left.\right|_{x=0}=0$.

Therefore, it is demonstrated that the $3 \mathrm{D}$ shape of the water surface can be reconstructed using the sampling moiré method combined with the phase-shifting technique.

\section{Sub-pixel sampling moiré method}

The closer the sampling spacing is to the initial fringe spacing, the smaller the phase error will be. However, the moiré patterns described above are formed by integer pixel sampling. Due to the limitation of camera resolution, it is difficult to obtain the fringe spacing with exactly an integer. In the case where the fringe pitch is not an integral multiple of the number of phase-shifting steps, there will be a phase error in the sampling moire patterns. This is a typical problem for the sampling moiré method.

To solve this problem, we propose a sub-pixel sampling moiré method. The basic principle is to interpolate the fringe pattern at the very beginning, then sample it with a sub-pixel pitch to form moiré patterns. In this way, the phase of the multiple moiré patterns formed by the sampling continuously covers a complete fringe pitch, thereby improving the accuracy of the phase shift.

The bicubic spline interpolation [31] is used to interpolate the fringe image captured. The sub-pixel intensity of the fringe patterns can be expressed as [30]:

$$
\begin{aligned}
& I^{*}\left(x^{\prime}, y^{\prime}\right)=g\left(x^{\prime}-x_{i-1}, y^{\prime}-y_{j-1}\right)=\sum_{m=0}^{3} \sum_{n=0}^{3} \\
& a_{m n}^{i j}\left(x^{\prime}-x_{i-1}\right)^{m}\left(y^{\prime}-y_{i-1}\right)^{n}
\end{aligned}
$$

where $x_{i-1}<x^{\prime}<x_{i}$ and $y_{j-1}<y^{\prime}<y_{j}, i$ and $j$ are indexes of the integer-pixel position of the fringe intensity matrix, $a_{m n}^{i j}$ is coefficients depend on the gray value and distance of the 16 pixels around this point.

Sampling in image $I^{*}(x, y)$ with a sub-pixel pitch $M^{*}$, the $Q$ th phase-shift images can be described as:

$$
I_{k}^{*}\left(x^{\prime}, y^{\prime}\right)=I_{a}^{*}\left(x^{\prime}, y^{\prime}\right)+I_{b}^{*}\left(x^{\prime}, y^{\prime}\right) \cos \left[\varphi_{m}\left(x^{\prime}, y^{\prime}\right)+2 \pi \frac{k^{*}}{M^{*}}\right]
$$

where $k^{*}=(0,1,2 \ldots, Q-1) \times \frac{M^{*}}{Q}$ is the order of the phaseshifted images.

Then, combining equations (3)-(7), the 3D topography of the transparent liquid can be reconstructed with improved accuracy.

\section{Sensitivity analysis}

According to equations (1)-(4), it indicates that the height components $\mathrm{d} h(x, y)$ and $h(x, y)$ are related to the in-plane

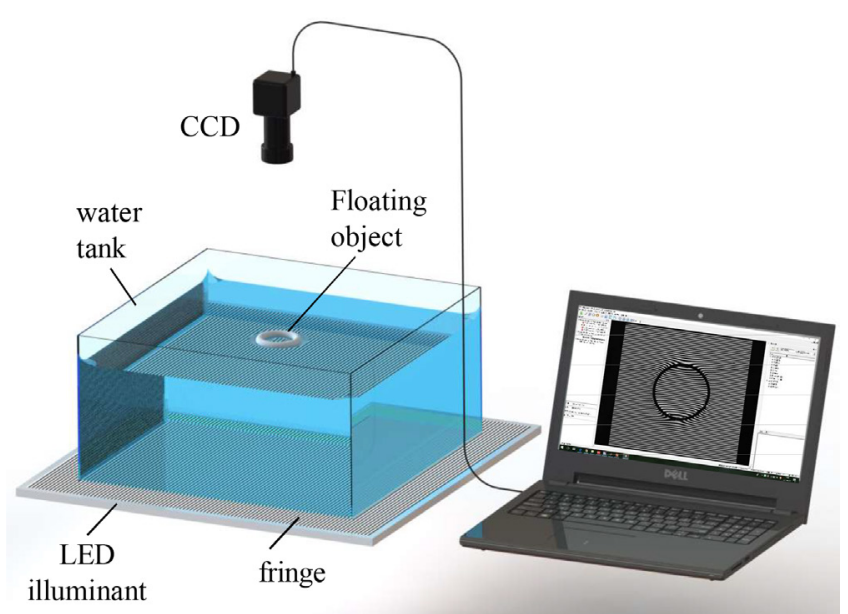

Figure 3. Experiment diagram showing the measurement of a liquid surface topography using monoscopic fringe transmissometry.

displacement $S(x, y)$. Therefore, the sensitivity of this new technique in measuring liquid topography is mainly dependent on the sensitivity of sampling moiré method. For method that calculated displacement using five-step phase-shifting technique, the sensitivity of displacement is normally better than $1 \%$ of the fringe pitch, which means that if the fringe pitch is $1 \mathrm{~mm}$ used in the measurement, the sensitivity of the in-plane displacement can theoretically reach $10 \mu \mathrm{m}$. Assuming that the initial height of liquid $H$ is $10 \mathrm{~mm}$, the theoretical sensitivity of the liquid height measurement is better than $0.40 \mu \mathrm{m}$ theoretically using a megapixel camera.

\section{Experiments}

\section{Experimental set-up}

To verify the feasibility and robustness of the developed monoscopic fringe transmissometry, we conducted verification test on the liquid surfaces deformed by floating $3 \mathrm{D}$ printed characters, as shown in figure 3.

The LED illuminant was placed on a vibration-isolating table, and was leveled to be horizontal. The black-transparent fringe film with a frequency of 5 line $\mathrm{mm}^{-1}$ was placed on the LED illuminant. Then the cuboid water tank filled with clean water $(H=26.5 \mathrm{~mm})$ was placed on the film. The CCD camera (MER-500-7UM with camera model IMAVISION, computer $14 \mathrm{~K}$ ) was installed right above the water tank and was connected to a computer. The lens was adjusted to focus on the fringe film with an image scale of 25 pixels $\mathrm{mm}^{-1}$.

\section{Experimental procedure}

In this paper, three polymer characters 'B I T' floating on the water surface were used to create a complex liquid surface topography. These characters were produced by a 3D printer (JGAURORA Z-603S) with hollow shapes, as show in figure 4(a). The material for printing the characters is polylactic acid (PLA), with a density about $1.2-1.3 \mathrm{~g} \mathrm{~cm}^{-3}$. Although this material is denser than water, the printed small characters can still float on the water surface due to the surface 
(a)

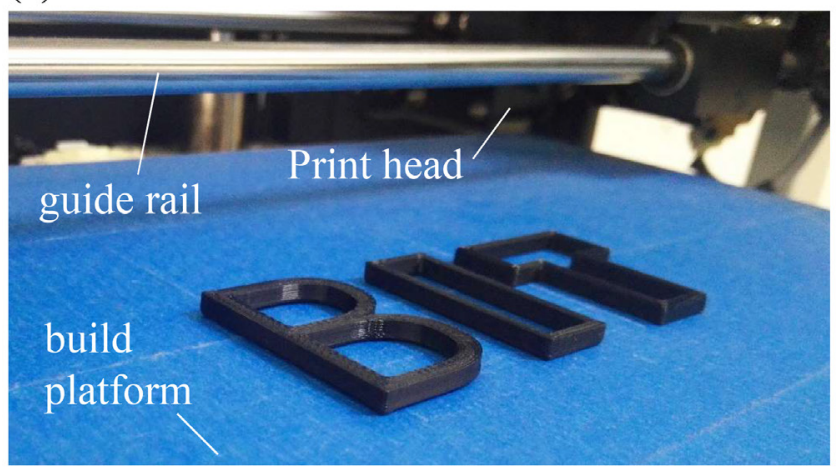

(b)
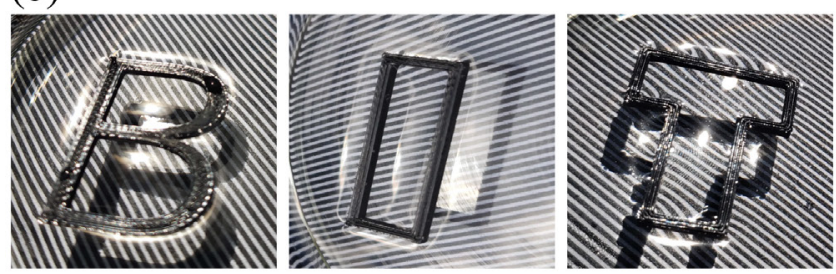

(c)

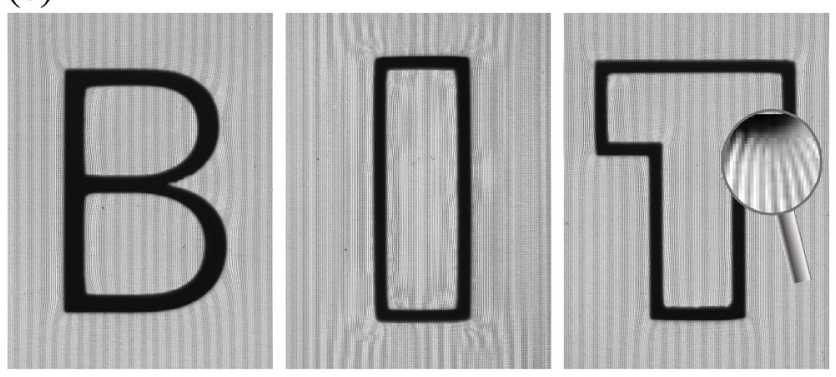

Figure 4. Experimental images. (a) Characters 'B I T' produced by the 3D printer. (b) An visually oblique view of liquid surface deformation caused by the floating characters. (c) Distorted fringe patterns captured from right above of the water surface. Inset: enlarged view of the distorted fringes next to the boundary of the character ' $\mathbf{T}$ '.

tension and the buoyancy, and cause slight deformation of the surface. This deformation reveals the competition between surface tension, buoyancy and gravity, so its measurement is very important.

The characters were gently and horizontally placed on the water surface so that each of them floated individually on the surface, as shown in figure 4(b). The special hollow shape of the characters deformed the water surface and divided it into some separate areas. After illuminating the fringe film with the LED illuminant, the CCD camera captured fringe patterns with the floating characters, as shown in figure 4(c). Then the characters were taken away to capture the reference images under the same conditions. The side profiles of floating characters were also captured from the side of the tank, which was used to demonstrate the developed method.

\section{Results and discussion}

\section{Image processing}

The fringes distorted by the floating characters shown in figure 4(c) were due to the refraction of light on the deformed water surface. They have fringe spacing of 4.91 pixel. The fringe patterns were proceeded using sub-pixel sampling moiré method as section 2 described. Interpolating both deformed and undeformed fringe images by a spline calculation. A series of moiré images can be obtained by starting sampling at different positions with the same spacing. Therefore, a sampling spacing of 4.91 pixels was set in the calculation. Moiré patterns were generated by sampling the fringe patterns at a 4.91 pixel spacing, starting at every 0.98 pixel step, and then interpolating by a spline interpolation, as shown in figure 5(a). In the process of the sampling and interpolating to obtain moiré patterns, moiré fringes were not formed in the region of the characters due to blocking of light.

To extract the phase component from the moire patterns, we employed the five-step phase-shifting technique [32] to proceed the patterns.

The discontinuity of the moire fringes did not affect the extraction of the raw phase (i.e. wrapped phase) because the phase-shifting was a point calculation process. The true phase can be obtained by phase unwrapping [33, 34], which started synchronously at several different positions of each region to ensure correctness. Small noise in the images can be eliminated by a cubic spline interpolation and averaging filter smoothing algorithm. The region of the characters was interpolated with a certain value to obtain the whole unwrapped phase. Finally, the displacement field was obtained using equations (2)-(4), as shown in figure 5(b).

As can be seen in figure 5(b), the displacement in the region covered by the characters was missed or greatly distorted. Herein, we extract the outline of the characters in the fringe patterns to extract the corresponding character images, as shown in figure 5(c). They were used as a mask to cover the messy displacement field to transform it into reasonable value, as shown in figure 5(d). Since the character coverage region had no displacement value, it was displayed as blank. Therefore, the morphology calculation program will know how to stop the iteration calculation and how to start again.

\section{Water surface reconstruction}

After the displacement field was obtained, the liquid surface reconstruction was then carried out. The operations were as follows:

(1) The displacement data or phase data were iterated point by point for each row to calculate the corresponding height information based on equation (1). The iteration was applied with initial height value of zero.

(2) When one row was completed, the calculation went to the next row until the whole data were traversed.

(3) The iteration did not apply to discontinuous displacement in the figure. In this case, the iteration stopped on one side of the character and then started again on the other side of its boundary, and the initial height value was as same as the stopped side.

(4) Repeated steps (1)-(3) until the whole filed height was calculated.

Figure 6 shows the reconstructed water surface caused by the floating characters. In the island surrounded by the outlines 
(a)

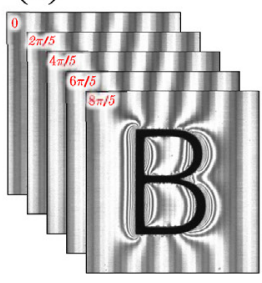

(c)

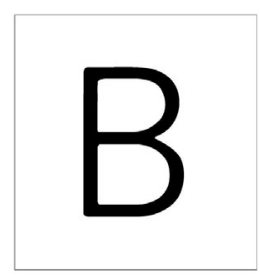

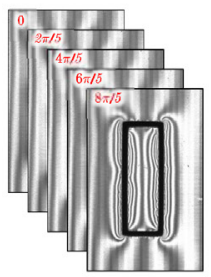
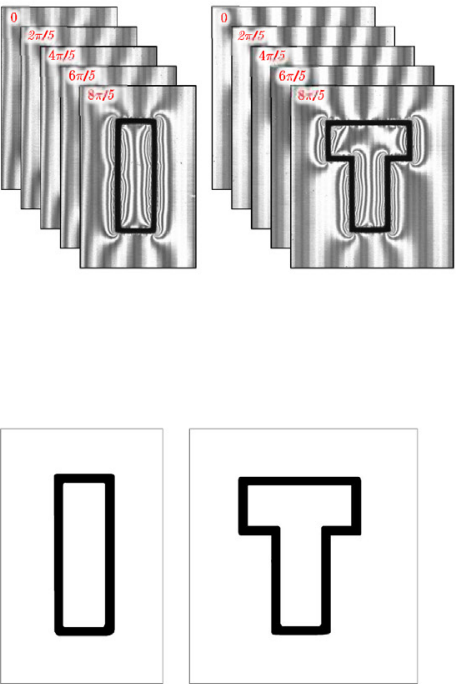

(b)

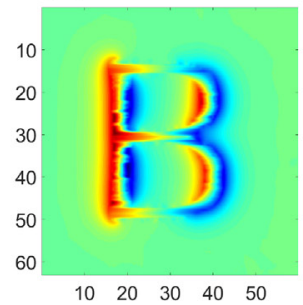

(d)

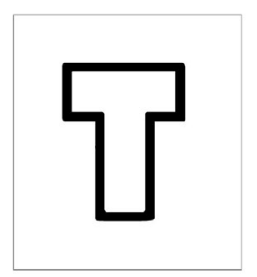

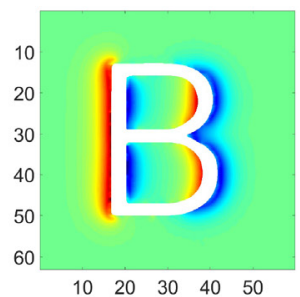

Unit: mm
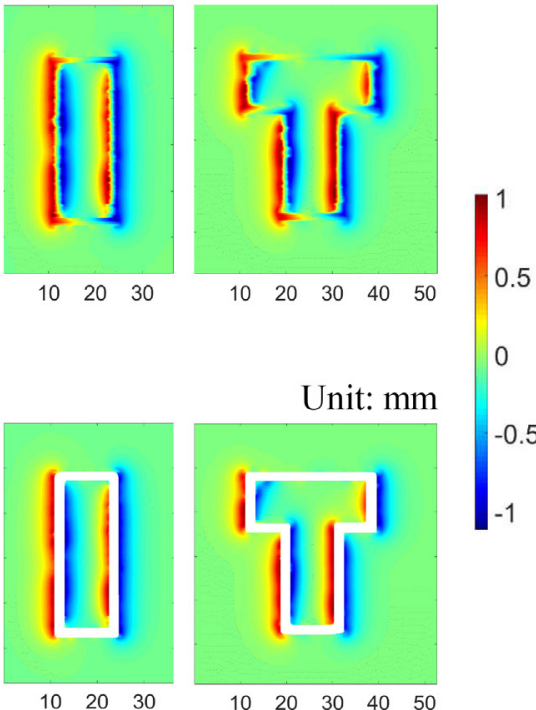

Figure 5. (a) A series of sampling moiré patterns. (b) Raw displacement fields. (c) Extracted masks for eliminating the displacement in the regions covered by the characters. (d) Reformed displacement fields.

of the characters, the shape of the liquid surface topographies looks like a smooth mountain, where the minimum is below the horizontal.

Using this method, we also measured the liquid surface deformation caused by a paper clip floating on the water surface supported by surface tension $[35,36]$ and buoyancy. The 3D shape of the deformed water surface caused by a floating paperclip was reconstructed by the same processes, as shown in figure 7.

\section{Comparison with existing method}

To compare with the existing method, the transmission-virtual grating method [20] was used to measure the liquid surface topography with a character 'B' floating. Since the basic light path of the method proposed in this paper is similar to the transmission-virtual grating method, the initial image shown in figure 4(c) can be used to measure the surface deformation with the transmission-virtual grating method. The topography result is shown in figure 8 .

Figure 8(a) is the displacement field, and figure 8(b) is the reconstructed topography of the outer contour. By comparing the surface morphology results of the monoscopic fringe transmissometry method shown in figure 8(b) without internal information with those of the transmission virtual grating method shown in figure 6 , the proposed method can obtain a more comprehensive surface morphology.

\section{Error analysis}

When a character ' $\mathrm{B}$ ' is floating on water surface, the side profiles of deformed liquid surface at the same position of the

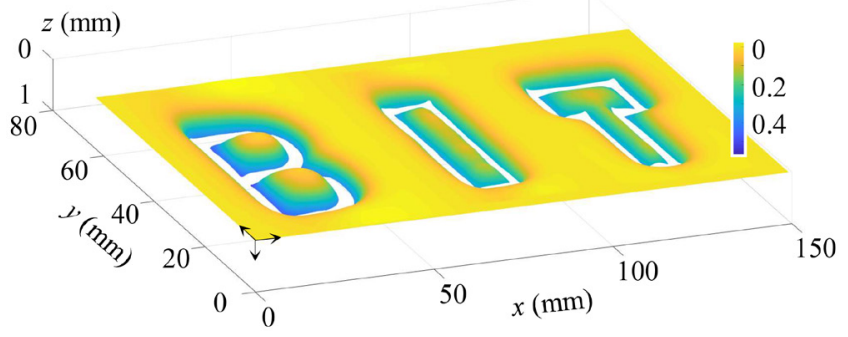

Figure 6. The 3D shape of the water surface ' $\mathbf{B} \mathbf{I} \mathbf{T}$ ' reconstructed by the monoscopic fringe transmissometry.

largest diameter is compared by the three methods, as shown in figure 9. The Pearson correlation coefficient [37] between the proposed method and the captured line is of $0.9997(0$ is worst and 1 or -1 is best). The Pearson correlation coefficient between the proposed method and the grating method is of 0.9993. The Pearson correlation coefficient between the grating method and the captured line is of 0.9994 , which means the measurement result is well consistent with the actual situation.

The liquid levels at the lowest point of the meniscus measured by the proposed method and read in $2 \mathrm{D}$ photography were $0.43 \mathrm{~mm}$ and $0.45 \mathrm{~mm}$, respectively. The relative error was: $\left|e_{r}\right|=\left|\frac{h^{*}-h}{h^{*}}\right|=4.36 \%$. Since the density of PLA was only slightly greater than that of water, this means that the surface tension and buoyancy of water played an important role in the floating of characters and cause tiny deformation of the water surface. This increased the measurement difficulty and error to some extent. In the case of larger surface deformation or topography of transparent objects and the application of higher water levels in the measurement, the relative error can be further reduced. 
(a)

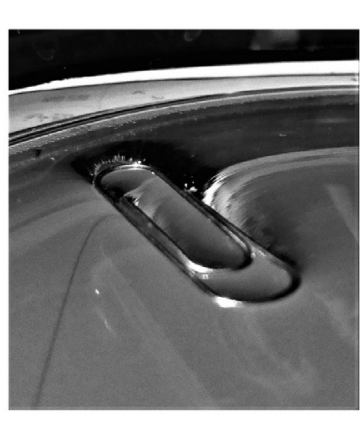

(b)

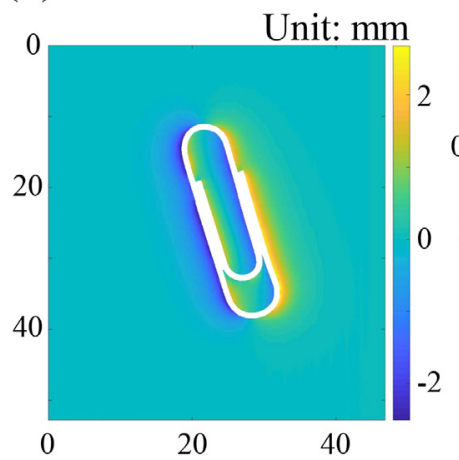

(c)

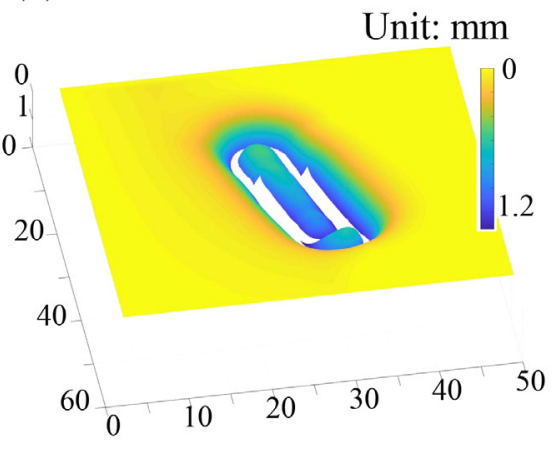

Figure 7. The reconstruction of the deformed water surface caused by floating paperclip by the monoscopic fringe transmissometry. (a) An visually oblique view of liquid surface deformation caused by the floating characters; (b) displacement filed; (c)3D morphology of the reconstructed surface.

(a)

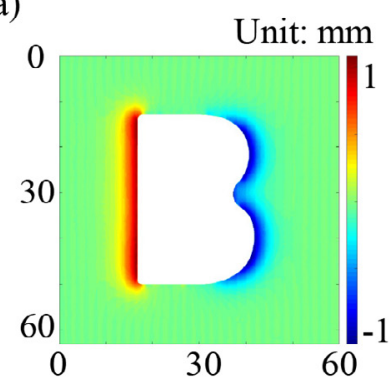

(b)

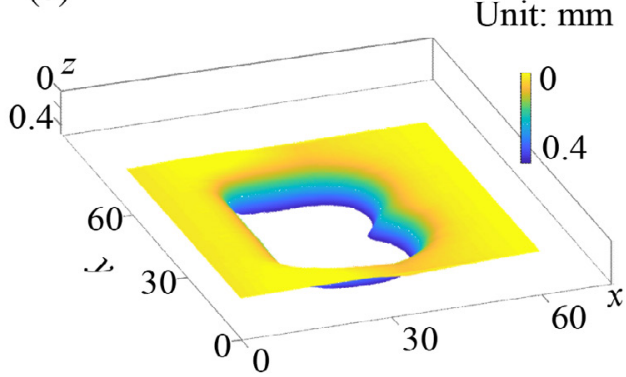

Figure 8. Measurement of deformed liquid surface topography with character 'B' by transmission-virtual grating method: (a) displacement field and (b) surface topography.

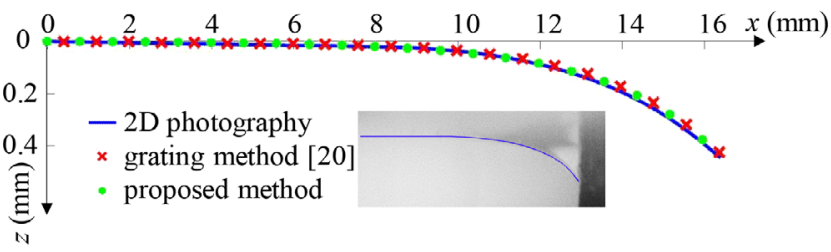

Figure 9. Comparison of the side profiles of the surfaces reconstructed by sub-pixel sampling moiré method and the grating method and the direct captured surface of character ' $\mathbf{B}$ '.

\section{Conclusions}

In this paper, we report the monoscopic fringe transmissometry for measuring 3D shape of complex liquid surfaces. The developed technique only requires projecting a single fringe pattern from the bottom of the liquid and capturing a single image from above. Due to the light refraction, the captured fringe pattern is deformed as the surface of the liquid deforms. We then propose the sub-pixel sampling moiré method to process the fringe pattern with high precision, which is combined with the phase-shifting technique to calculate the displacement field, and then reconstruct the liquid surface deformation based on the derived equations. If the exiting light is blocked by the floating object, it will cause loss or discontinuity of the fringe, resulting in difficulty in image processing and data loss in the region of the object. In this paper, we circumvent this dilemma using a digital mask extracted from the outline of the floating object. The experimental results of the liquid surface deformation caused by the characters made of PLA show that the results of the developed technique are in good agreement with the actual measurement results.

The main advantages of the developed technique are:

(1) It only requires very simple apparatus and only needs to capture a single deformed image to obtain the phase value, which means that the method can be widely used, especially in the dynamic measurement of liquid surfaces.

(2) Due to the applications of sub-pixel resolution sampling moiré method and the phase-shifting technique, the developed technique has extremely high sensitivity and precision.

(3) The developed technique can be used to measure complex topography of discontinuous transparent liquid surface.

It should be noted that there is also disadvantage of the proposed method. It has limitations in the measurement of liquid with varying density, which will be the focus of our future work.

\section{Acknowledgments}

This work was financially supported by the National Natural Science Foundation of China (Grant Nos. 11572041 and 11702299). 


\section{Notes}

The authors declare no competing financial interest.

\section{ORCID iDs}

Menglin Zhong (ㅇ https://orcid.org/0000-0002-5139-9352

Xianfu Huang 으 https://orcid.org/0000-0002-5859-8135

\section{References}

[1] Liu J-L, Feng X-Q and Wang G-F 2007 Buoyant force and sinking conditions of a hydrophobic thin rod floating on water Phys. Rev. E 76066103

[2] Hu D L, Chan B and Bush J W M 2003 The hydrodynamics of water strider locomotion Nature 424 663-6

[3] Bush J W M and Hu D L 2006 Walking on water: biolocomotion at the interface Ann. Rev. Fluid Mech. 38 339-69

[4] Teixeira M A C, Arscott S, Cox S J and Teixeira P I C 2015 What is the shape of an air bubble on a liquid surface Langmuir 31 13708-17

[5] Dickinson M 2003 Animal locomotion: how to walk on water Nature 424 621-2

[6] Caponigro M A and Eriksen C H 1976 Surface film locomotion by the water strider Gerris remigis say Am. Midland Naturalist 95 268-78

[7] Peterson L D, Crawley E F and Hansman R J 1989 Nonlinear fluid slosh coupled to the dynamics of a spacecraft AIAA J. 27 1230-40

[8] Veldman A E P, Gerrits J, Luppes R, Helder J A and Vreeburg J P B 2007 The numerical simulation of liquid sloshing on board spacecraft J. Comput. Phys. 224 82-99

[9] Martel C, Nicolás J A and Vega J M 1998 Surface-wave damping in a brimful circular cylinder J. Fluid Mech. 360 213-28

[10] Vreeburg J P B 2005 Spacecraft maneuvers and slosh control IEEE Control Syst. 25 12-6

[11] Rebouillat S and Liksonov D 2010 Fluid-structure interaction in partially filled liquid containers: a comparative review of numerical approaches Comput. Fluids 39 739-46

[12] Celebi M S and Akyildiz H 2002 Nonlinear modeling of liquid sloshing in a moving rectangular tank Ocean Eng. 29 1527-53

[13] Gómez-Goñi J, Garrido-Mendoza C A, Cercós J L and González L 2013 Two phase analysis of sloshing in a rectangular container with volume of fluid (VOF) methods Ocean Eng. 73 208-12

[14] Pal N C, Bhattacharyya S K and Sinha P K 2003 Nonlinear coupled slosh dynamics of liquid-filled laminated composite containers: A two dimensional finite element approach J. Sound Vib. 261 729-49

[15] Liu D and Lin P 2009 Three-dimensional liquid sloshing in a tank with baffles Ocean Eng. 36 202-12

[16] Hinsch K D 1978 Holographic interferometry of surface deformations of transparent fluids Appl. Opt. 17 3101-7
[17] Matsuda K, Watanabe S and Eiju T 1985 Real-time measurement of large liquid surface deformation using a holographic shearing interferometer Appl. Opt. 24 4443-7

[18] Moisy F, Rabaud M and Salsac K 2009 A synthetic Schlieren method for the measurement of the topography of a liquid interface Exp. Fluids 46 1021-36

[19] Huang L, Ng C S and Asundi A K 2011 Dynamic threedimensional sensing for specular surface with monoscopic fringe reflectometry Opt. Express 19 12809-14

[20] Liu Z, Huang X and Xie H 2013 A novel orthogonal transmission-virtual grating method and its applications in measuring micro 3D shape of deformed liquid surface $O p t$. Lasers Eng. 51 167-71

[21] Liu S, Liu Z-W and Shi W-X 2014 A source for the excellent floating ability of a water strider Chin. Phys. Lett. 31106801

[22] Shi W, Huang X and Liu Z 2014 Transmission-lattice based geometric phase analysis for evaluating the dynamic deformation of a liquid surface Opt. Express 22 10559-69

[23] Yang Y, Liu Z-W, Shi W-X and Huang X-F 2015 Accurate measurement of nonlinear liquid sloshing AIAA J. 53 771-9

[24] Liu Z, Guo J, Shi W, Huang X and Xie H 2015 Transmissionspeckle correlation for measuring dynamic deformation of liquid surface Opt. Lasers Eng. 65 110-6

[25] Tang H, Dong H and Liu Z 2017 Study on dynamic deformation synchronized measurement technology of double-layer liquid surfaces Opt. Lasers Eng. 98 205-16

[26] Zhang H, Liu Z, Wen H, Xie H and Liu C 2016 Subset geometric phase analysis method for deformation evaluation of HRTEM images Ultramicroscopy 171 34-42

[27] Ri S, Fujigaki M and Morimoto Y 2010 Sampling moiré method for accurate small deformation distribution measurement Exp. Mech. 50 501-8

[28] Fujigaki M and Morimoto Y 2011 Dynamic shape and strain measurements of rotating tire using a sampling moiré method Opt. Eng. 50101506

[29] Creath K 1985 Phase-shifting speckle interferometry Appl. Opt. 24 3053-8

[30] Huntley J M 1998 Automated fringe pattern analysis in experimental mechanics: a review J. Strain Anal. Eng. Des. 33 105-25

[31] Carl D B 1962 Bicubic spline interpolation J. Math. Phys. $41212-8$

[32] Stoilov G and Dragostinov T 1997 Phase-stepping interferometry: five-frame algorithm with an arbitrary step Opt. Lasers Eng. 28 61-9

[33] Gierloff A J J 1987 Phase unwrapping by regions Curr. Dev. Opt. Eng. II 8182

[34] Saldner H O and Huntley J M 1997 Temporal phase unwrapping: application to surface profiling of discontinuous objects Appl. Opt. 36 2770-5

[35] Zhao Y P 2012 Physical Mechanics of Surfaces and Interfaces (Beijing: Science Press)

[36] Huang X, Dong H, Liu Z and Zhao Y 2019 Probing micro-newton forces on solid/liquid/gas interfaces using transmission phase-shift Langmuir 35 5442-7

[37] Adler J and Parmryd I 2010 Quantifying colocalization by correlation: the Pearson correlation coefficient is superior to the Mander's overlap coefficient Cytometry A 77 733-42 